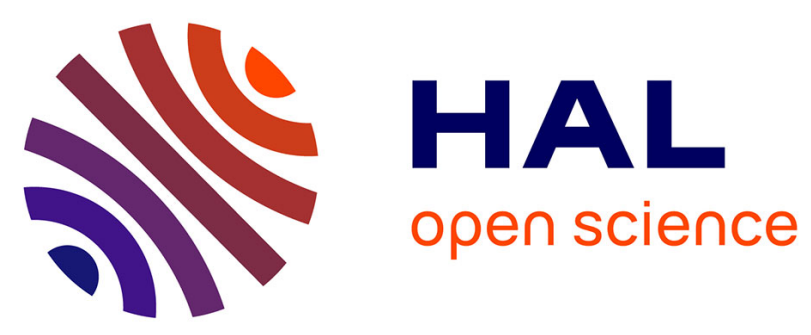

\title{
Collision-free workspace of parallel mechanisms based on an interval analysis approach
}

Mohammad Hadi Farzaneh Kaloorazi, Mehdi Tale Masouleh, Stéphane Caro

\section{To cite this version:}

Mohammad Hadi Farzaneh Kaloorazi, Mehdi Tale Masouleh, Stéphane Caro. Collision-free workspace of parallel mechanisms based on an interval analysis approach. Robotica, 2017, 35 (8), pp.1747-1760. 10.1017/S0263574716000497 . hal-01721328

\section{HAL Id: hal-01721328 \\ https://hal.science/hal-01721328}

Submitted on 19 Apr 2018

HAL is a multi-disciplinary open access archive for the deposit and dissemination of scientific research documents, whether they are published or not. The documents may come from teaching and research institutions in France or abroad, or from public or private research centers.
L'archive ouverte pluridisciplinaire HAL, est destinée au dépôt et à la diffusion de documents scientifiques de niveau recherche, publiés ou non, émanant des établissements d'enseignement et de recherche français ou étrangers, des laboratoires publics ou privés. 


\title{
Collision-free workspace of parallel mechanisms based on an interval analysis approach MohammadHadi FarzanehKaloorazi $\dagger^{*}$, Mehdi Tale Masouleh $\ddagger$ and Stéphane Caro§
}

\author{
$\dagger$ CoRo, École de Technologie Supérieure, Montréal, Quebec, Canada \\ $\ddagger$ Human-Robot Interaction Laboratory, Faculty of New Sciences and Technologies, University of \\ Tehran,Tehran,Iran.E-mail:m.t.masouleh@ut.ac.ir \\ $\S C N R S-I R C C y N$, UMR 6597, 1 rue de la Nö̈, 44321 Nantes, France. \\ E-mail: stephane.caro@irccyn.ec-nantes.fr
}

\begin{abstract}
SUMMARY
This paper proposes an interval-based approach in order to obtain the obstacle-free workspace of parallel mechanisms containing one prismatic actuated joint per limb, which connects the base to the end-effector. This approach is represented through two cases studies, namely a 3-RPR planar parallel mechanism and the so-called 6-DOF Gough-Stewart platform. Three main features of the obstacle-free workspace are taken into account: mechanical stroke of actuators, collision between limbs and obstacles and limb interference. In this paper, a circle(planar case)/spherical(spatial case) shaped obstacle is considered and its mechanical interference with limbs and edges of the end-effector is analyzed. It should be noted that considering a circle/spherical shape would not degrade the generality of the problem, since any kind of obstacle could be replaced by its circumscribed circle/sphere. Two illustrative examples are given to highlight the contributions of the paper.
\end{abstract}

KEYWORDS: Parallel mechanism; Collision-free workspace; Mechanical interference; Interval analysis.

\section{Introduction}

Parallel mechanisms (PMs) are known to be more precise and able to carry heavier loads than the serial manipulators. ${ }^{1,2}$ Besides several advantages, they are restricted by their limited workspace. Therefore, in practice, the presence of an obstacle inside their workspace should be taken into consideration in order to alleviate this limitation. Obtaining the obstacle-free workspace of PMs leads to have a conservative workspace for which all actions inside the aforementioned workspace are free of collision. Furthermore, obtaining the obstacle-free workspace is a definite asset in path planning and obstacle avoidance while controlling PMs.

The problems of path planning and obstacle avoidance have been frequently investigated in the literature. ${ }^{3}$ In ref. [4], $\mathrm{Zi}$ et al. considered the possible collision of a cooperative cable-driven parallel robot for multiple mobile cranes and used the sensor technology in order to avoid obstacles. Laliberte et al. in ref. [5] calculated the motion of the manipulator using the velocity inversion of a redundant manipulator, which optimizes the distance to obstacles. The algorithm includes joint limit constraints, collision detection and heuristics for the solution of typical difficult cases, thereby leading to a high success rate. Brooks et al. proposed an efficient algorithm to generate collision-free paths for a manipulator with five or six revolute joints. Yang et al. investigated dynamic collision-free trajectory generation in a non-stationary environment, using biologically inspired neural network approaches. Brocks and Khatib ${ }^{6}$ represented elastic strip framework which

\footnotetext{
* Corresponding author. E-mail: hamidfarzane88@gmail.com
} 
enables the execution of a previously planned motion in a dynamic environment for robots with many degree of freedom (DOF). Khatib et al. ${ }^{7}$ presented developments of models, strategies and algorithms dealing with a number of autonomous capabilities that are essential for robot operations in human environments. These capabilities include integrated mobility and manipulation, cooperative skills between multiple robots, interaction ability with humans and efficient techniques for real-time modification of collision-free paths. In ref. [8], Komainda and Hiller presented a concept for motion control of redundant manipulators in a changing environment. In ref. [9], Jiménez et al. described a general approach to cover all distance computation algorithms, hierarchical object representations, orientation-based pruning criteria and space partitioning schemes. In ref. [10], Wenger and Chedmail illustrated the collision-free workspace of serial manipulators. Caro et al., in ref. [11], introduced a new method, based on numerical constraint programming, in order to compute a certified enclosure of the generalized aspects. In ref. [12], the collision-free workspace of a planar PM is investigated. This paper aims at extending the latter study by proposing a general concept to be applicable to 6-DOF PMs, such as Gough-Stewart platform.

Most of the approaches presented in the literature are case dependent and cannot be generalized and extended to other cases. In ref. [13], the workspace of 3-RRR PMs has been investigated. Even for this particular PMs, the approach could be applied upon considering some assumptions, where in the latter paper, the mechanisms should be symmetric. It should be noted that in the foregoing paper, the workspace is only investigated without considering mechanical interferences and obstacles within the workspace.

The mathematical framework used in this paper is based on interval analysis. ${ }^{14-16}$ Interval analysis is a reliable method to evaluate functions and is used frequently in the field of robotics. ${ }^{17}$ An interval variable of $[x]=[\underline{x}, \bar{x}]$ is a set of all real numbers from the lower bound, $\underline{x}$, to the upper bound, $\bar{x}$. More details about the application of interval analysis in kinematic investigation of robotic mechanical system are discussed in Section 2 and in ref. [14].

In the context of application of interval analysis in investigating the kinematic properties of PMs, several papers have been published. Most of them dealt with solving the Forward Kinematic Problem $(\mathrm{FKP})^{18}$ and obtaining the workspace. ${ }^{16,19,20}$ Merlet, in ref. [21], investigated the workspace of PMs. Several papers have been published in the context of obtaining the singularity-free workspace using geometrical approach, ${ }^{22}$ and using interval analysis ${ }^{16}$ in which the novelty is about demonstrating the state of the art of applying interval analysis in solving the foregoing problems. In ref. [23], authors used interval analysis in order to investigate the orientation workspace of a parallel kinematic machine. Merlet, in ref. [24], proposed an algorithm that enables one to determine almost all the geometries of a simplified Gough platform whose workspace should include an arbitrary set of poses. There have been few study conducted on analyzing the workspace of PMs by considering mechanical interference and also in the presence of obstacle. This is a definite asset both in design and control of PMs, which is the main concern of this paper.

Few studies have been conducted on obtaining the collision-free workspace of PMs and this is mainly due to the fact that this mechanisms have complex kinematic expressions comparing to their serial counterpart. Interval analysis has been used more in solving the FKP and in obtaining theoretical workspace, i.e., workspace in which mechanical interference and obstacle are not taken into account. Some papers worked on obtaining the collision-free workspace of cable-driven PMs which is out of the scope of the present paper. In the latter papers, the approach is mostly based on numerical techniques in which all the configurations and poses of the end-effector (EE) are tested in order to obtain configurations for which collision may occur. ${ }^{25}$ For instance, interval analysis has been used in investigating the workspace of cable-driven PMs. ${ }^{26}$

The main contribution of this paper deals with the use of interval analysis techniques in order to obtain the collision-free workspace of parallel manipulators. It should be noted that obtaining the collision-free workspace of robotic mechanical systems, PMs among others, is a very delicate task which is elusive to classical methods. Therefore, more elaborated methods should be used such as interval analysis techniques. To the best of author's knowledge, few studies have been conducted in this regard.

The remainder of this paper is organized as follows. First, the mathematical framework of interval analysis is broadly reviewed. Then, the concept of the algorithm developed to obtain the obstacle-free workspace and the corresponding pseudo-code are explained in detail. Finally, the results obtained for a 3-R $\underline{P R}$ and a 6-UPS PM are given and the corresponding obstacle-free workspaces are illustrated. 


\section{Interval Analysis and Mathematical Framework}

Several people had the idea to bound rounding errors with intervals: e.g. Dwyer (1951), ${ }^{27}$ Sunuga $(1958),{ }^{28}$ Warmus $(1956)^{29}$ and Wilkinson $(1980) .{ }^{30}$ However, interval mathematics has been widespread in the research community with Moore's book "Interval Analysis" in 1966. ${ }^{31}$ Moore's book transformed this simple idea into a viable tool for error analysis. Instead of merely treating rounding errors, Moore extended the use of interval analysis to bound the effect of errors from all sources, including approximation and errors in data. ${ }^{32}$ In the literature, interval analysis is regarded as a powerful numerical method to solve a wide range of problems such as, among others, circumventing round-off errors ${ }^{18}$ solving system of equations, optimization problem ${ }^{32}$ and proper workspace presentation, etc... ${ }^{17,32-34}$ Furthermore, interval analysis provides an interactive visualization in the progress of calculation which is a definite asset in $2 \mathrm{D}$ and $3 \mathrm{D}$ representations of manipulator workspaces. Recently, upon revealing some remarkable features of interval analysis, such as finding the solution of a problem within some finite domains and taking into account the numerical computer round-off errors, it has stimulated the interests of many researchers in the robotic community to deal with complicated problems such as FKP and inverse kinematic problem (IKP), ${ }^{18,35}$ calibration $^{36,37}$ and the determination of the singularity-free workspace of parallel manipulators. ${ }^{16,19}$

In the evolutionary techniques, the chance of being trapped in a local optimum is highly dependent of the initial population and initial search space. However, in the case of interval analysis, the only parameter to obtain the global optimum is to choose the proper search space. In order to compute the maximum singularity-free workspace of PMs and other kinematic properties, ${ }^{17,33}$ interval analysis entails following advantages: (a) Contrary to other tools, which would result in a lengthy computation process and may converge to a local optimum, interval analysis is not a black box, since it requires combination of heuristics and numerical concepts to be effective; (b) it allows us to find all the solutions with inequalities within a given search space, ${ }^{18,38}$ (c) for two and three-dimensional problems, it leads to see the evolution of the solutions and to monitor the procedure in order to have better insight to the problem; (d) it allows us to consider uncertainties in the model of the robot.

Interval analysis is a branch of mathematics that basically works with closed intervals instead of accurate numbers. An interval $[x]$ is a set of real numbers between two bounds and can be represented as follows:

$$
[x]=[\underline{x}, \bar{x}]=\{x \in \mathbb{R} \mid \underline{x} \leq x \leq \bar{x}\},(\underline{x} \leq \bar{x}),
$$

where $\underline{x}$ and $\bar{x}$ are lower bound and upper bound, respectively. All mathematical operations such as addition and multiplication can be performed on intervals. For instance, ${ }^{14}$

$$
\begin{aligned}
{[x]+[y] } & =[\underline{x}, \bar{x}]+[\underline{y}, \bar{y}]=[\underline{x}+\underline{y}, \bar{x}+\bar{y}], \\
{[x][y] } & =[\min (S), \max (S)], \quad S=\{\underline{x} \underline{y}, \underline{x} \bar{y}, \bar{x} \underline{y}, \bar{x} y\} .
\end{aligned}
$$

Moreover, a function of real numbers such as $f(x)$ can be evaluated as an interval from a given interval, $[x]$, which results in an interval $[f]=f([x])$. For example, for a monotonic function like $f(x)=x^{3}$,

$$
[f]=f([x])=[f(\underline{x}), f(\bar{x})]=\left[\underline{x}^{3}, \bar{x}^{3}\right] .
$$

The whole concept of interval analysis is based on bisecting a box (or a hyper-box in a higher dimensional space), called branch \& prune approach, ${ }^{39}$ upon considering some well-defined algebra on intervals and natural evaluations, in such a way that the latter box will converge toward the desired solution.

\section{Obstacle Avoidance Formulation via Geometrical Concept}

To the sake of understanding the proposed method, the latter is first fully described for 3-RPR PM. It should be noted that, without loss of generality, the obstacle is considered as a circle (planar case) or a sphere (spatial case) since any kind of obstacle could be replaced by its circumscribed circle or sphere. In order to avoid an obstacle, in general, the links of limbs and the edges of the EE are considered to be straight line segments. Moreover, all links can be considered to be perfect cylinder (rectangle in planar case). In the case of more complicated shape of a link, one should simplify 


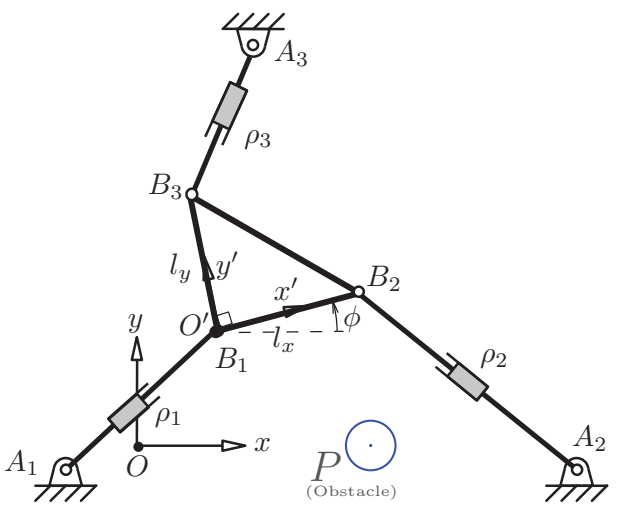

(a)

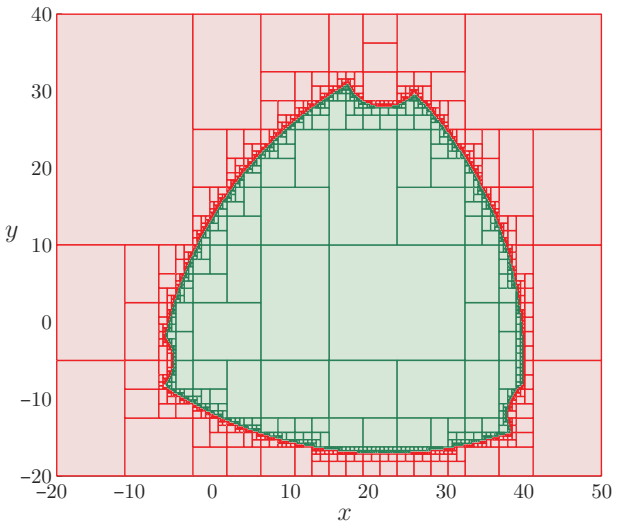

(b)

Fig. 1. (a) Schematic representation of a 3-RPR planar PM and a circle-shaped obstacle located at point $P$, $\rho_{\min }=5$ and $\rho_{\max }=50$. and (b) the constant-orientation workspace of a $3-\mathrm{RPR}$ planar PM for $\phi=\pi / 4$ via interval analysis, collisions are ignored. $A_{1}=\{-10,-5\}, A_{2}=\{50,-5\}$ and $\overline{A_{3}}=\{15,40\} ; x_{P}=20, y_{P}=0$ and $r_{P}=3 ; l_{x}=l_{y}=10$. Green boxes are inside and red boxes are outside the workspace.

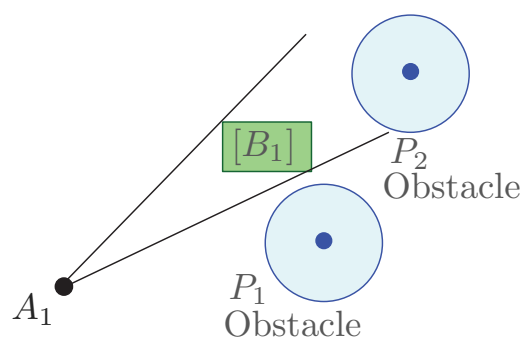

(a)

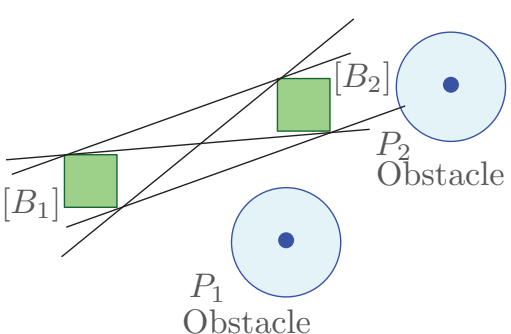

(b)

Fig. 2. Two types of interval lines. In both cases, there is no collision if the obstacle is located at either point $P_{1}$ or point $P_{2}$. (a) Interval line passing through one point, $A_{1}$, and one box, [ $B_{1}$ ]; link $A_{1} B_{1}$ in Fig. 1(a). (b) Interval line passing through two boxes, $\left[B_{1}\right]$ and $\left[B_{2}\right]$; edge $B_{1} B_{2}$ of the EE in Fig. 1(a).

it to a cylinder which contains all parts of the link. Even though it may cause to eliminate some non-collision part of the workspace, but it depends on the trade-off preference of the user to keep a balance between the computational time and precision of the result. This is a common approach to compute the constant-orientation workspace of parallel robots. In the case of 6-DOF PMs, since here is no human visualization for depicting six-dimensional space, thus one should fix the rotational DOF to have a graphical representation of the workspace.

As it can be observed in Fig. 1, in the case of planar PMs, the obstacle is a circle; $x_{P}$ and $y_{P}$ being the Cartesian coordinates of its center point and $r_{P}$ its radius. The problem of obtaining the obstacle-free workspace via interval analysis can be divided into two cases: the collisions between the obstacle and (a) a line passing through one point and one box (b) through two boxes. These two cases are depicted in Fig. 2. The first case is applicable for links that are connected to the fixed frame via a revolute joint; for instance, links $A_{1} B_{1}, A_{2} B_{2}$ and $A_{3} B_{3}$ in the 3-RPR planar PM shown in Fig. 1(a). The second case is more general and is applicable for those links which are the medial or distal links of the limb; there is not such a link in the 3-RPR planar PM. Moreover, the edges of the EE should be categorized in the second case, i.e., in the 3-RPR planar PM, $B_{1} B_{2}, B_{2} B_{3}$ and $B_{3} B_{1}$.

\subsection{Interval distance to obstacle}

A simple solution to obtain the obstacle-free workspace is to write the equation from the distance of a point to a line in the $2 \mathrm{D}$ space, which can be written as follows:

$$
\begin{gathered}
d=\sqrt{\left(\frac{x_{P}+m y_{P}-m c}{m^{2}+1}-x_{P}\right)^{2}+\left(m \frac{x_{P}+m y_{P}-m c}{m}+c-y_{P}\right)^{2}} \\
L: y=m x+c,
\end{gathered}
$$


Table I. Geometric parameters of a 3-RPR PM.

\begin{tabular}{rrrccrrr}
\hline$i$ & $x_{A_{i}}$ & $y_{A_{i}}$ & $\rho_{\min }$ & $\rho_{\max }$ & $x_{B_{i}^{\prime}}$ & $y_{B_{i}^{\prime}}$ & unit \\
\hline 1 & -10 & -5 & 5 & 50 & 0 & 0 & $\mathrm{~cm}$ \\
2 & 50 & -5 & 5 & 50 & 10 & 0 & $\mathrm{~cm}$ \\
3 & 15 & 40 & 5 & 50 & 0 & 10 & $\mathrm{~cm}$ \\
\hline
\end{tabular}

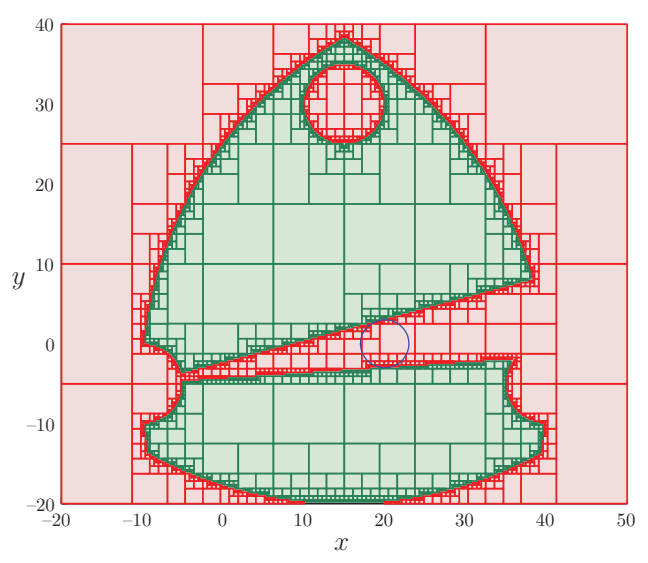

(a)

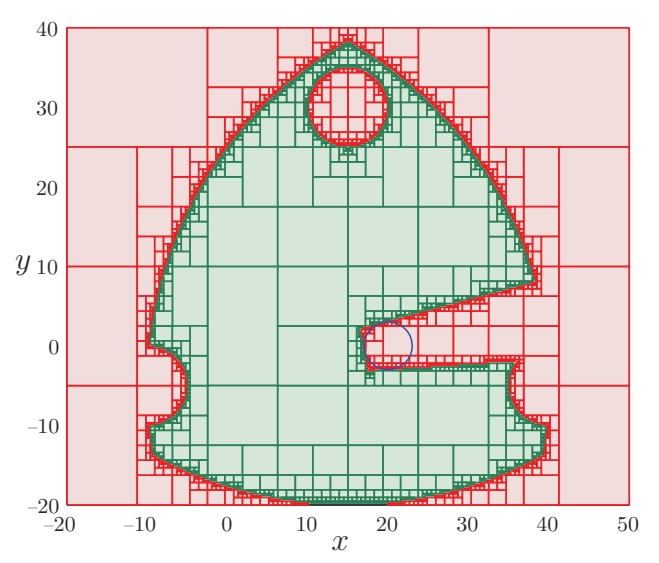

(b)

Fig. 3. The obstacle-free workspace of a 3-RPR planar PM shown in Fig. 1(a), for $\phi=0$, only considering the first limb $A_{1} B_{1}$. Green boxes are inside and red boxes are outside the obstacle-free workspace. (a) $A_{1} B_{1}$ as a line satisfying $[d]>r_{P}$. (b) $A_{1} B_{1}$ as a segment line satisfying $[d]>r_{P}$ and $\|A-[B]\|_{2}<\|A-P\|_{2}$.

where $d$ stands for the distance from point $P$ to line $L, x_{P}$ and $y_{P}$ are the $x$ and $y$ coordinates of the obstacle $P$, respectively, $m$ stands for the slope of the line and $c$ is a constant.

In the case of implementing an interval line using a point and a box, the first aforementioned case, one has

$$
m=\frac{y_{A}-\left[y_{B}\right]}{x_{A}-\left[x_{B}\right]}, \quad c=y_{A}-m x_{A},
$$

in which $x_{A}$ and $y_{A}$ stand for the coordinates of point $A,\left[x_{B}\right]$ and $\left[y_{B}\right]$ stand for the components of box $[B]$. These lines are referred to as the collision lines, which are assigned to the links of mechanisms and in some configurations may interfere with the obstacle.

\subsection{Collision of limbs with obstacle}

Resorting to interval functions, ${ }^{1}$ one can apply interval variables to Eq. (5) and obtain an interval of distances, $[d]$. For example, in the case of the 3-RPR PM shown in Fig. 1(a), having geometric parameters represented in Table I, for the first limb, the collision line passes through $A_{1}$, which is a fixed point, and a box in the search space of the interval algorithm. The task of the algorithm is to determine the distance from obstacle $P$ to the collision line. If $[d]>r_{P}$, i.e., the distance of all possible lines passing through the fixed point and the box under investigation will be higher than the obstacle radius, then the corresponding box will be fully inside the obstacle-free workspace. On the other hand, if $[d]<r_{P}$, the box will be fully outside of the aforementioned workspace. Eventually, if $0 \in[d]-r_{P}$ the box goes for further bisection. The result of the above procedure is illustrated in Fig. 3(a). It is noteworthy that the green circles in the figures correspond to the lower limits of the actuators.

\subsection{Collision of edges of EE with obstacle}

The procedure of obtaining the obstacle-free workspace is not complete yet. Indeed, the RPR limb should be regarded as a segment instead of a line. Therefore, if $\|A-[B]\|_{2}<\|A-P\|_{2}$, then $[B]$ does not interfere with the obstacle and should be a member of obstacle-free workspace, Fig. 3(b). 


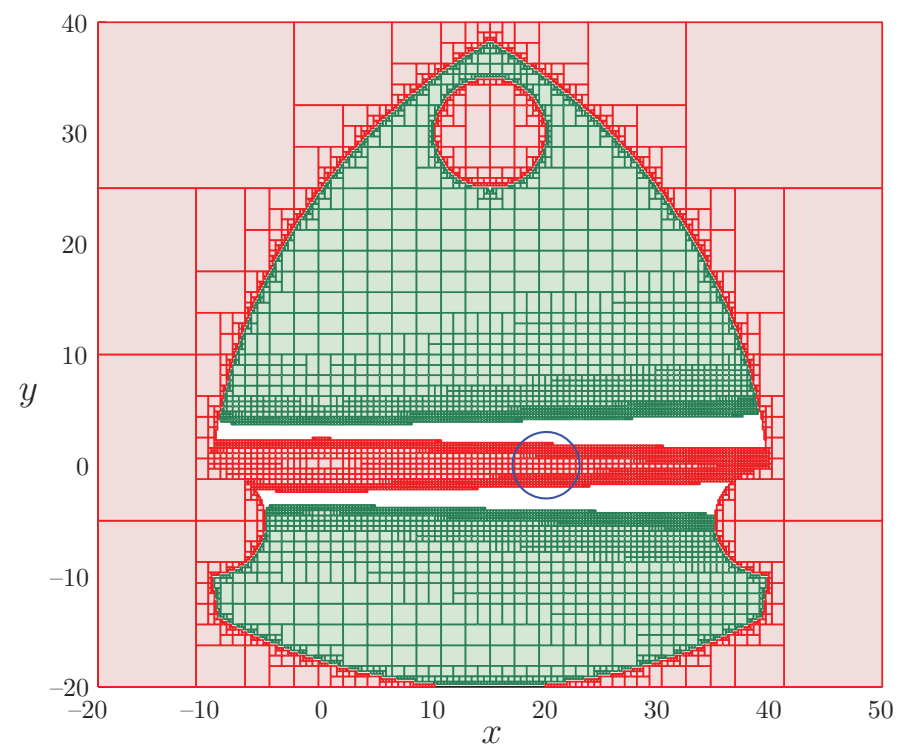

Fig. 4. The obstacle-free workspace, considering only edge $B_{1} B_{2}$ of EE, for $\phi=\pi / 4$. The result is undesirable due to interval blow up.

In addition to the limbs, the interference between the edges of the EE and the obstacle should also be taken into account. In the case of 3-DOF PMs, a simple EE can be regarded as a triangle. Therefore, its edges belong to those lines passing through two intervals, i.e., the second case. In this case, obtaining the distance from the obstacle to the line, using Eq. (5), leads to a very time consuming and inefficient process. Figure 4 represents the obstacle-free workspace in the case that only the collision of the edge $B_{1} B_{2}$ with the obstacle is taken into consideration. The result shown in Fig. 4 is not a desirable result. The white area, which shows unassigned boxes, comparing to Fig. 3(b), is a vast area. Furthermore, there are too many red boxes and green boxes in the middle of the workspace. From the previous results, we expected that the number of boxes would be lower. This shows that computational load of these boxes is high. The latter problem is known as blow up. Blow up is a common phenomenon in interval analysis, which appears when some intervals are multiplied together and when degree of complexity of the interval function becomes high. ${ }^{32}$ Hence, a geometrical methodology is proposed in order to eliminate those parts of the workspace for which edges of the EE collide with the obstacle. Since the workspace is represented for a constant orientation, the slope of the interval line is constant. In other words, by determining the dimensions (width and height) and position of $\left[B_{1}\right]$, the position and dimensions of $\left[B_{2}\right]$ are known. The result of considering a constant slope for the interval line passing through two boxes is represented in Fig. 5. In the latter figure, the edge $B_{1} B_{2}$ is regarded as a line and the workspace is obtained for a constant orientation of $\phi=\pi / 4$. However, the result is not complete because $B_{1} B_{2}$ should be regarded as a segment line.

In order to find the obstacle-free workspace, only considering $B_{1} B_{2}$, a geometrical approach, based on the fact that the workspace is obtained for a constant orientation, is combined with interval analysis. For the sake of better understanding, first a simple example is discussed here. The example can be observed in Fig. 6. Assuming that the problem consists in obtaining a shape within the workspace which should be subtracted from the full workspace. In the constant orientation case, the slope of edge $B_{1} B_{2}$ of the EE is constant, in this example $\phi=\pi / 4$. The workspace consists of the set of locations that point $B_{1}$ can reach in the fixed frame, because the moving frame is attached to the EE in $B_{1}$. As it can be observed from Fig. 6, the shape is a rounded rectangle. A rounded rectangle is a shape which is generated by sweeping the center of a circle along a segment line. It is a rectangle having a rounded cap instead of right angle. An example of rounded rectangle is shown in Fig. 6. The next step is to define the shape using geometrical reasoning. By considering three geometrical constraints for the center of obstacle circle, the shape can be obtained: The center is subject to (a) the line passing through point $P$ and of slope $\phi$ (b) the interval $\left[x_{C}\right]$ along $x$-component (c) interval $\left[y_{C}\right]$ 


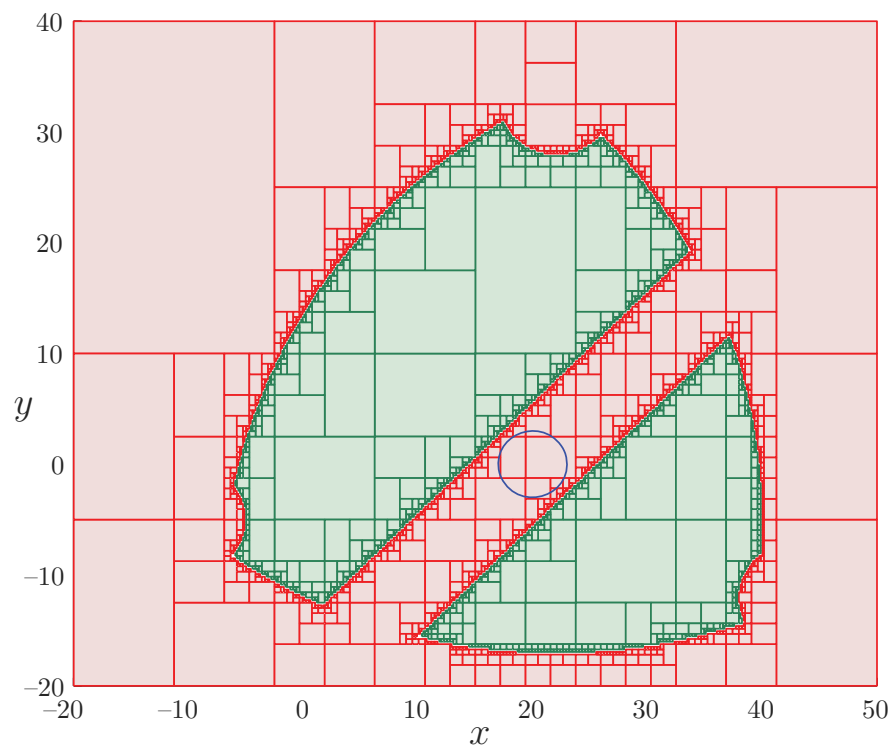

Fig. 5. The obstacle-free workspace, considering only edge $B_{1} B_{2}$ of EE, for $\phi=\pi / 4$. This is a modified result of Fig. 4. A better solution is represented in Fig. 7(a).

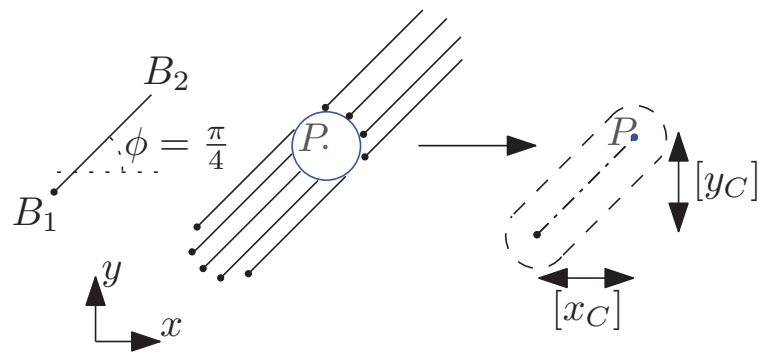

Fig. 6. Technique to find the shape that should be subtracted from the full workspace. This rounded rectangle is the shape within the workspace that shows the locations of $B_{1}$ where edge $B_{1} B_{2}$ collides with the obstacle circle.

along $y$-component. For the case study of this paper, one has

$$
\begin{aligned}
& {\left[x_{C}\right]=x_{P}-\left[0, \overline{B_{1} B_{2}} \cos \phi\right]} \\
& {\left[y_{C}\right]=y_{P}-\left[0, \overline{B_{1} B_{2}} \sin \phi\right] .}
\end{aligned}
$$

Figure 7(a) illustrates the results of the foregoing methodology, considering only the collision of edge $B_{1} B_{2}$ with the obstacle, for $\phi=0$. In this figure, a rounded-end rectangle inside the workspace is eliminated. Upon considering all the three edges of the EE, Fig. 7(b) represents the obstacle-free workspace of the 3-RPR planar PM for $\phi=\pi / 4$.

Algorithm 1, in the appendix, represents the pseudo-code of the introduced method. It is based on a branch and prune algorithm. ${ }^{39}$ Note that $f$, which indicates the number of actuated joint of the mechanism, is 3 in the case of planar PM. In the upcoming section, we will extend the algorithm to a higher DOF mechanism, i.e. 6-UPS PM and in that case $f=6$. There is another variable which is indicated by $g$, referring to the dimension of the box. In the case of planar PM, it is 2 and in the case of spatial PM it is 3. In lines 5-7, the position of the three distal joints is determined as intervals in the fixed frame. In line 9 , for all limbs, the distance from the distal to the proximal joints, i.e., the length of the actuator, is evaluated in order to be checked in line 15 . In line 10 , $\operatorname{Line}(A, B)$ is a function which creates a collision line that passes through points $A$ and $B$. Since the second argument is an interval $\left[B_{i}\right]$, hence the collision line is an interval line. Distance $(L, P)$ computes the Euclidean distance from point $P$ to line $L$. Therefore, $\left[d_{i}\right], i=1,2,3$, are intervals of possible distances from 


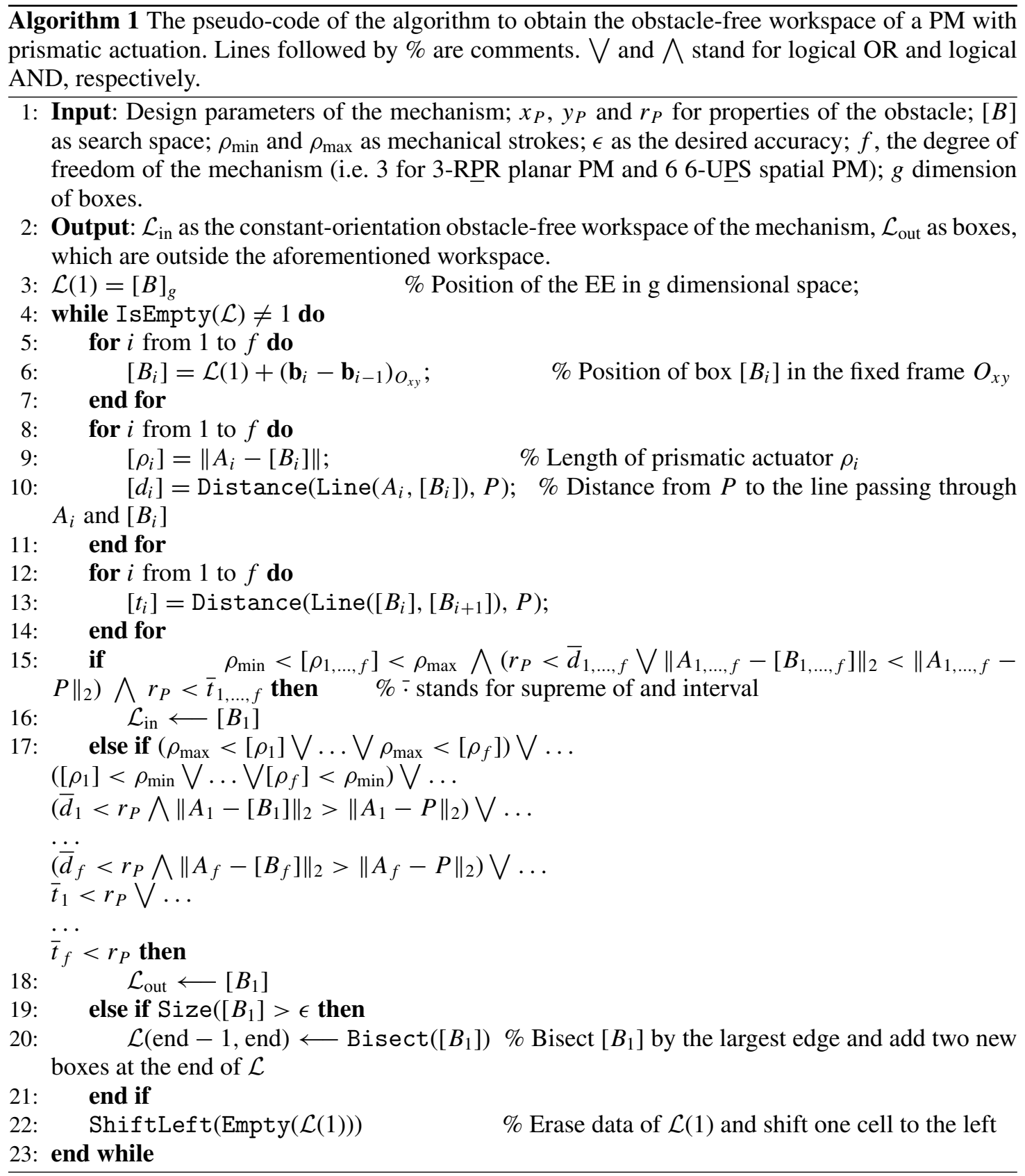

$P$ to the corresponding line of the $i$ th actuator. Lines 12-14 are interval lines passing through two intervals. Line 15 is the general if-clause for which if all $\left[\rho_{i}\right]$ are in the acceptable range and if all $\left[d_{i}\right]$ distances from $P$ to the collision lines are higher than $r_{P}$, then the box under study will be a member of the obstacle-free workspace, $\mathcal{L}_{\text {in }}$. There is an extra condition to ascertain that if the distance from the box $\left[b_{i}\right]$ to the fixed point $A_{i}$ is lower than the distance from $P$ to $A_{i}$, hence the box should be inside the obstacle-free workspace. On the other hand, if only one of the aforementioned criteria is violated, then the box will be moved to the outer boxes list, $\mathcal{L}_{\text {out }}$. In line 19 , if the box under study is partially inside the obstacle-free workspace and at least one of its dimensions is still larger than the desired precision, then it will be bisected by the largest edge (line 20) and two new boxes will be added at the end of list $\mathcal{L}$. In line $22, \operatorname{ShiftLeft}(\operatorname{Empty}(\mathcal{L}(1)))$, the first column of list $\mathcal{L}$ will be erased and all other elements of $\mathcal{L}$ will be moved one cell back to fill the gap. In other words, in each loop of the while-clause one box from list $\mathcal{L}$ will be investigated. In line 22, it is known that the aforementioned box is either located inside, outside or on the boundaries of the desired workspace; 


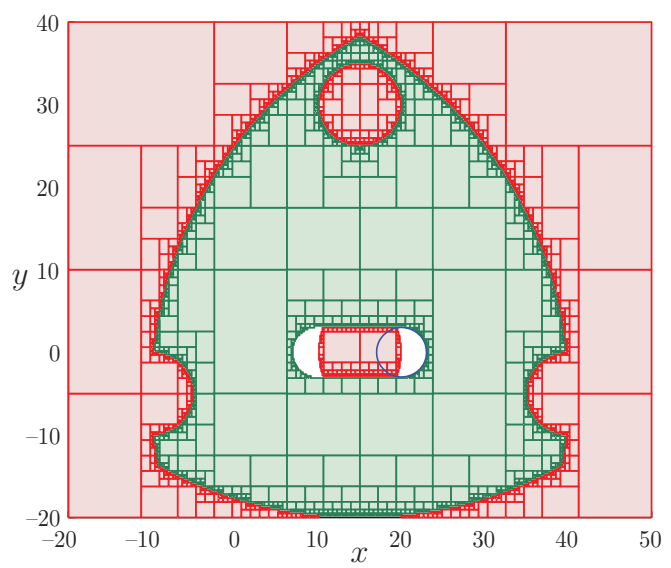

(a)

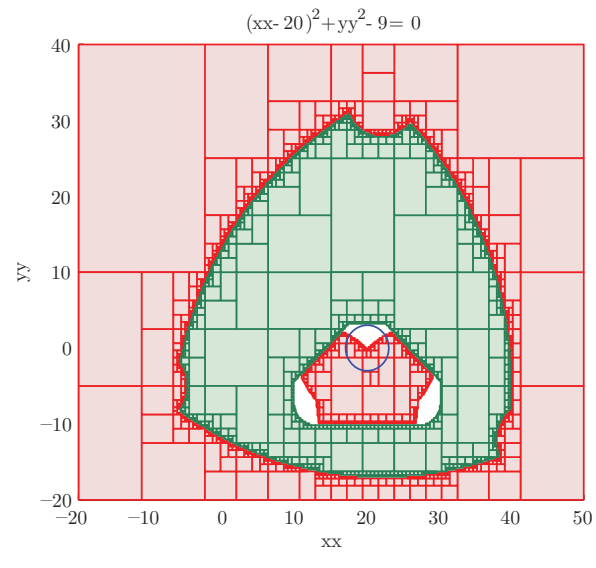

(b)

Fig. 7. The obstacle-free workspace of the mechanism shown in Fig. 1(a) by considering interval segment lines passing through two intervals. Green boxes are inside and red boxes are outside the obstacle-free workspace. (a) The case for which only $B_{1} B_{2}$ collides with the obstacle, $\phi=0$. (b) Regarding all edges of the EE, i.e. $B_{1} B_{2}$, $B_{2} B_{3}$ and $B_{3} B_{1}, \phi=\pi / 4$

therefore, there is no need to keep the box and erasing it will help the computer to free up memory. The algorithm continues until the prescribed precision is reached.

\section{Results for 3-RPR PM}

So far, an interval-based method to obtain the obstacle-free workspace of planar PMs, for a 3-RPR planar PM as a case study, has been introduced and the results of obstacle-free workspace, by considering only the collisions of one limb with its environment, Fig. 3(b), and only considering the collision of the edges of the EE, Fig. 7(b), are depicted. The next step is to put together all obstacle-free workspaces of all limbs. Figure 8(a) represents the obstacle-free workspace of the mechanism for a prescribed orientation, $\phi=\pi / 4$, by considering the collisions of all limbs with the obstacle. It should be noted that in Fig. 8(a), the obstacle is only inside the collision space of the first limb and as it can be observed, for the second and third limbs, the obstacle is located outside the corresponding red boxes. The latter is due to the fact that the represented workspace is depicted in the fixed coordinate frame and since a constant orientation of the EE is considered, thus other limbs should be translated into the fixed coordinate frame via the transformation of the moving frame. For instance, in the case of the 3-RPR planar PM for $\phi=\pi / 4$, if we move the obstacle along the vector of $-\left\{l_{x} \cos (\pi / 4), l_{y} \sin (\pi / 4)\right\}^{T}$, which is the negative of position of joint $B_{2}$ represented in the moving frame, the obstacle will be located in the red boxes caused by considering the collisions of the second limb and the obstacle.

The final step to obtain the obstacle-free workspace is to merge the obtained results and intersect them, i.e., considering the limb collisions, Fig. 8(a), and all edges of the EE, Fig. 7(b), with the obstacle. The final result is illustrated in Fig. 8 and as it could be expected, the obstacle divides the workspace into three separate parts, which are not connected to each other. The obtained obstacle-free workspace can be used in obstacle avoidance problems. As it is obtained via interval analysis, for all paths, whose points are located inside the obstacle-free workspace, it is guaranteed that the paths are free of obstacle collision.

The proposed interval-based method to obtain the obstacle-free workspace for planar PMs can be readily extended to more complicated and spatial PMs. Indeed, one can solve the IKP and use the obtained equations to determine the interval position of all joints and define the aforementioned segment lines to obtain the distances to the obstacle. It takes approximately $3 \mathrm{~min}$ to compute the obstacle-free workspace of the 3-RPR planar PM shown in Fig. 1(a), precision of $10^{-6}$ percent of initial search space, with a $2 \mathrm{GHz}$ processor, using INTLAB 6 toolbox. In the case of higher DOF mechanisms having more links, collision computation of each limb will be added into the computational time. 
Table II. Geometric parameters of the 6-UPS Spatial PM under study (all lengths are given in mm).

\begin{tabular}{lcrrrrr}
\hline $\mathrm{i}$ & 1 & 2 & \multicolumn{1}{c}{3} & \multicolumn{1}{c}{4} & \multicolumn{1}{c}{5} & \multicolumn{1}{c}{6} \\
\hline$x_{a_{i}}$ & 92.58 & 132.58 & 40.00 & -40.00 & -132.58 & -92.58 \\
$y_{a_{i}}$ & 99.64 & 30.36 & -130.00 & -130.00 & 30.36 & 99.64 \\
$z_{a_{i}}$ & 23.10 & 23.10 & 23.10 & 23.10 & 23.10 & 23.10 \\
$x_{b_{i}}$ & 30.00 & 78.22 & 48.22 & -48.22 & -78.22 & -30.00 \\
$y_{b_{i}}$ & 73.00 & -10.52 & -62.48 & -62.48 & -10.52 & 73.00 \\
$z_{b_{i}}$ & -37.10 & -37.10 & -37.10 & -37.10 & -37.10 & -37.10 \\
$\rho_{i_{\min }}$ & 454.5 & 454.5 & 454.5 & 454.5 & 454.5 & 454.5 \\
$\rho_{i_{\max }}$ & 504.5 & 504.5 & 504.5 & 504.5 & 504.5 & 504.5 \\
\hline
\end{tabular}

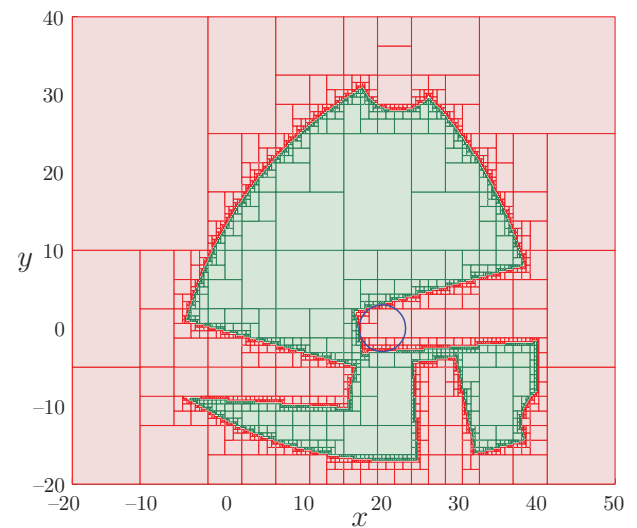

(a)

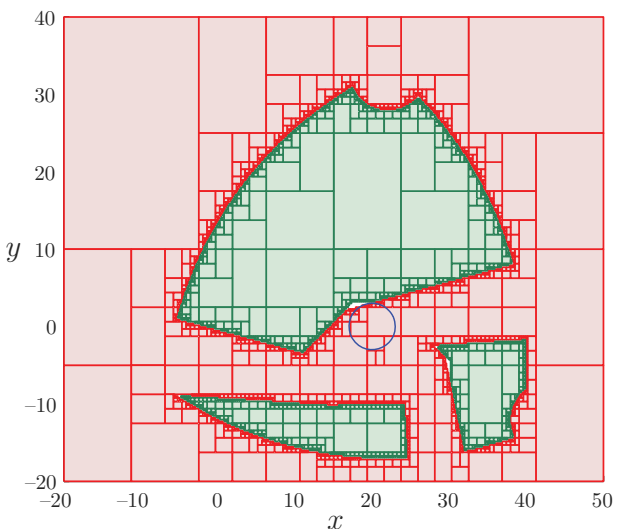

(b)

Fig. 8. The obstacle-free workspace of the 3-RPR planar PM shown in Fig. 1 , for $\phi=\pi / 4$, (a) considering limb collision only (b) final result considering all collisions. Green boxes are inside and red boxes are outside the obstacle-free workspace, respectively. (a) Only limbs collisions are taken into account, $\phi=\pi / 4$. (b) Intersection of all obstacle-free workspaces including all limbs and all edges of the EE.

For the sake of simplicity, the workspace of the mechanisms studied in this paper is represented for a constant orientation. This simplification does not affect the generality of the method, because it can be done for different orientations and represent a higher dimension collision-free workspace (3D workspace in case of planar mechanism for which $z$-axis is the orientation of the EE about $z$-axis).

\section{Results for a 6-UPS PM}

In this section, the aforementioned method is applied to obtain the obstacle-free workspace of a 6-UPS spatial PM. The proposed method is free from dimensional restriction and it can be applied to PM with more than two DOF, by only paying the computational time cost. A 6-UPS spatial PM is shown in Fig. 9(a) and its geometric parameters are represented in Table II. As it can be observed from Fig. 9, a sphere obstacle, $P$, is located inside the workspace of the mechanism. The workspace of the mechanism is obtained by using interval analysis, regardless of the obstacle, Fig. 9(b). ${ }^{17,38}$ The workspace is depicted considering mechanical stroke of prismatic joints and spherical and universal joint limits.

The main advantage of using this method is that it could be readily extended to 6-DOF; in fact, it is a feature of the proposed method. Algorithm 1 can be directly used to determine the obstacle-free workspace of the 6-UPS spatial PM. By choosing the number of DOF $f$ and the dimension of the box $g$, the algorithm computes the collision-free workspace of the corresponding PM, i.e., 3-RPR or 6-UPS. In line 6, the positions of all vertexes of the EE are obtained. Other lines of the algorithm is the same as before, by having in mind that the command Line creates a spatial line in the current case. Such 3D interval lines are illustrated in Fig. 10. 


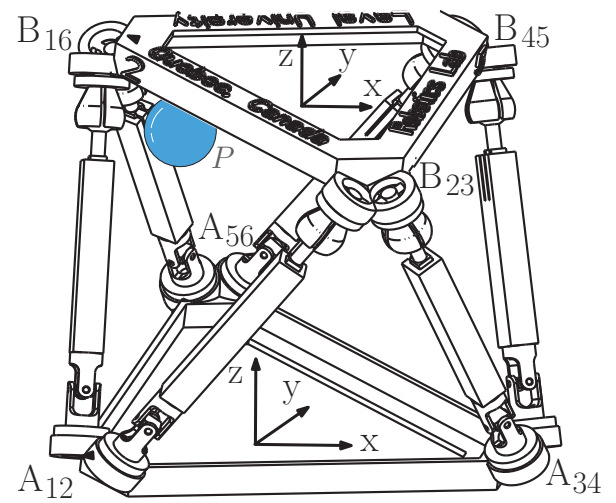

(a)

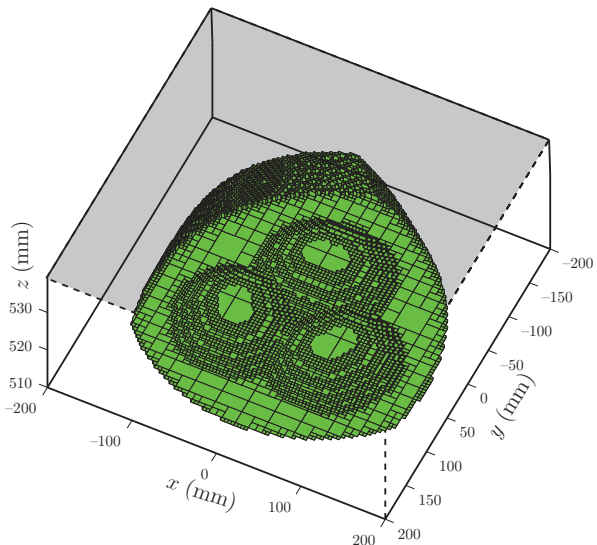

(b)

Fig. 9. (a) 6-UPS spatial PM and (b) the corresponding workspace considering actuation stroke, via interval analysis. $P=[100,0,500], r_{P}=10$. (a) A 6-UPS spatial PM and a sphere obstacle $P .{ }^{19}$ (b) The sliced constant-orientation workspace of a 6-UPS spatial PM, via interval analysis.

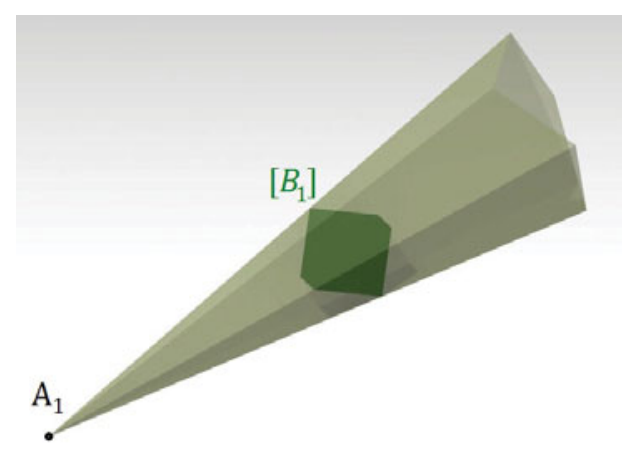

(a)

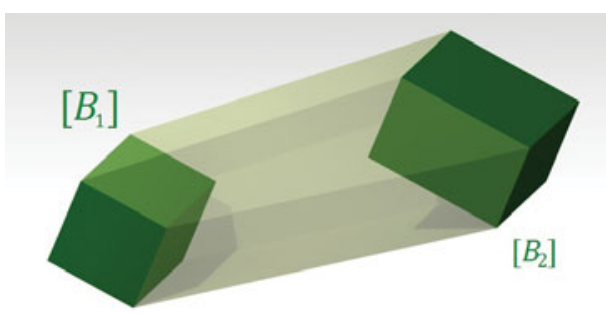

(b)

Fig. 10. Two types of interval lines in case of 3D workspace. (a) Interval line passing through one point, $A_{1}$, and one 3D box, $\left[B_{1}\right]$; link $A_{12} B_{16}$ in Fig. 9(a). (b) Interval line passing through two 3D boxes, $\left[B_{1}\right]$ and $\left[B_{2}\right]$; edge $B_{16} B_{23}$ of the EE in Fig. 9(a).

In the case of a spatial PM, corresponding interval lines are derived from 3D expressions, and the distance from line to the obstacle is expressed as follows:

$$
d=\frac{\left\|\left(\mathbf{x}_{2}-\mathbf{x}_{1}\right) \times\left(\mathbf{x}_{1}-\mathbf{x}_{0}\right)\right\|}{\left\|\mathbf{x}_{2}-\mathbf{x}_{1}\right\|},
$$

in which, $\mathbf{x}_{1}$ and $\mathbf{x}_{2}$ are two points on the line, in our case, positions of the $\mathrm{U}$ joint and $\mathrm{S}$ joint. Moreover, $\mathbf{x}_{0}$ stands for the center point of the obstacle.

Considering the limited workspace of the 6-UPS spatial PM, only two limbs of the mechanism clash with the obstacle, which are $A_{12} B_{16}$ and $A_{56} B_{16}$. By pursuing the same procedure, as introduced in the previous section for 3-RPR planar PM, the obtained workspace could be computed in which only the collisions of one limb with the obstacle is considered, Fig. 11. The workspace is shown in two views, namely, top view, Fig. 11(a), and sectioned isometric view, Fig. 11(b). In this case, a conical part of the workspace is removed.

The final workspace of the mechanism, while considering the collisions of one limb with the obstacle, is represented in Fig. 12. Since the workspace is obtained for a constant orientation and the workspace of the 6-UPS spatial PM is very limited, the moving platform will not collide with the obstacle. The workspace is in a constant orientation because there is no graphical representation for a workspace in a dimension higher than three. In Fig. 12, two views of the obstacle-free workspace is 


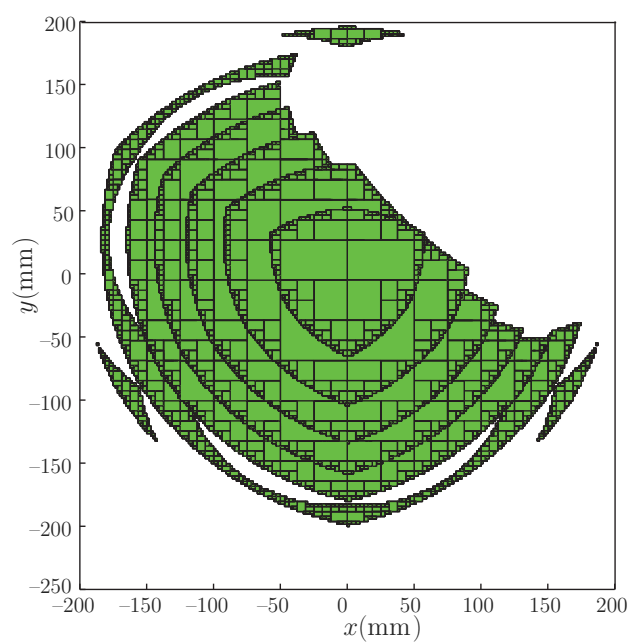

(a)

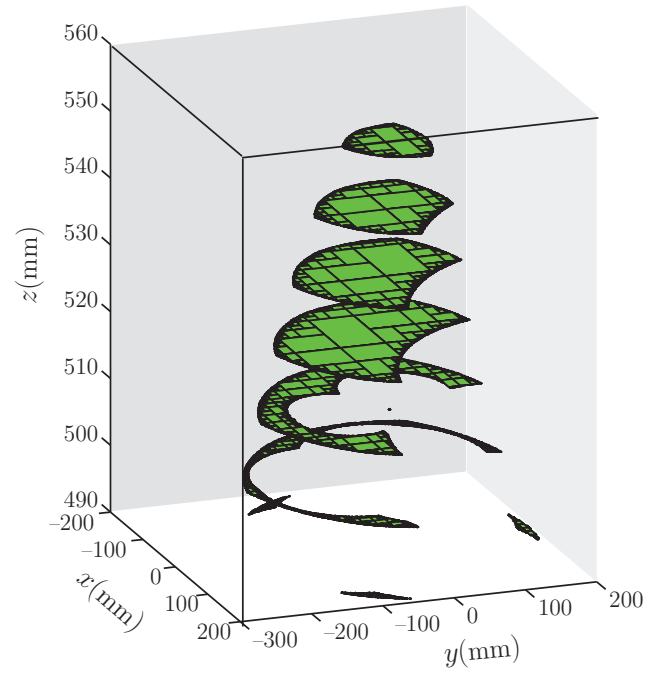

(b)

Fig. 11. Obstacle-free workspace of a 6-UPS Spatial PM, considering the collisions of one limb with the obstacle. (a) Top view (b) Sectioned isometric view.

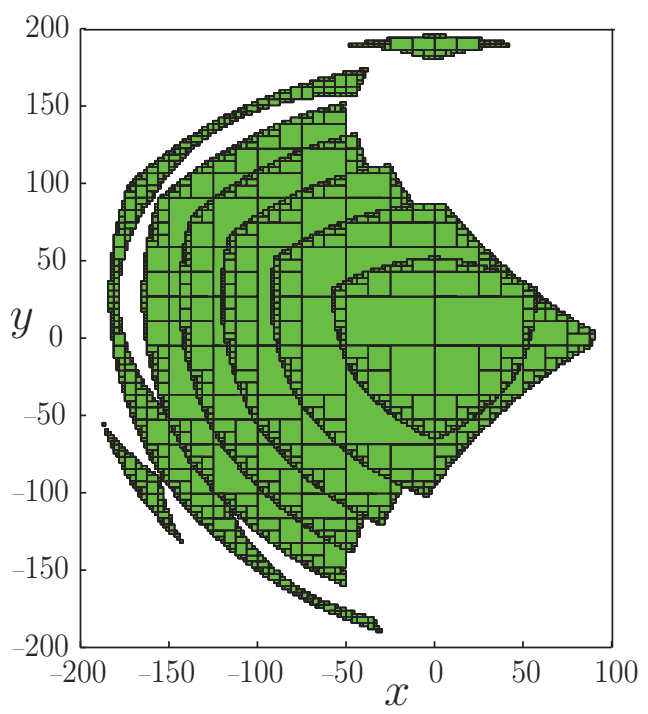

(a)

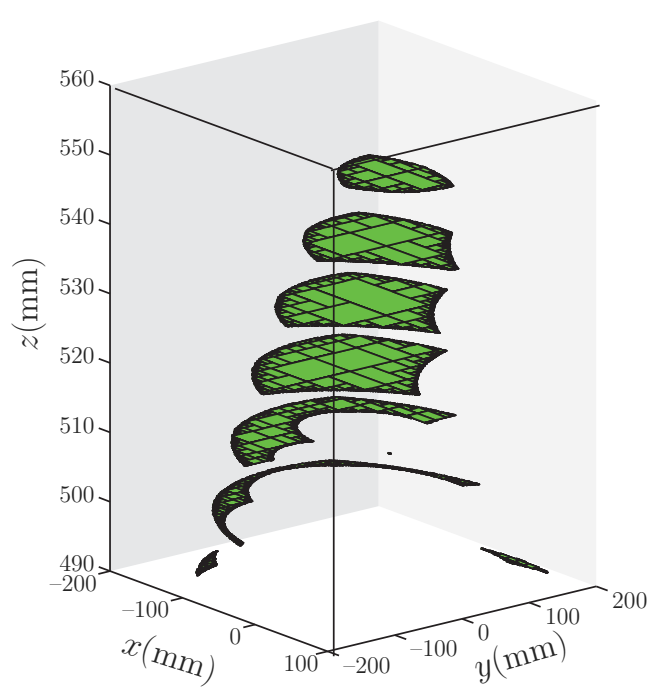

(b)

Fig. 12. Obstacle-free workspace of a 6-UPS Spatial PM, considering the collisions of all limbs with the obstacle, for the constant orientation of the EE at reference configuration, i.e. 0 for all three Euler angles. (a) Top view (b) Sectioned isometric view.

represented, which are top view, Fig. 12(a), and sectioned isometric view, Fig. 12(b). In the case that the obstacle is bigger than the one considered here, other limbs may collide with it and there will be more subtracted conical area in the workspace.

Computational time and required memory of the process is highly dependent of the desired precision. It can be improved by choosing a proper initial search box to avoid unnecessary computation of the area outside of the workspace. Moreover, a symmetric and simple design of the robot may lead to a lower computing time. For this case study, using a $2 \mathrm{GHz}$ CPU and $4 \mathrm{~GB}$ of ram and INTLAB toolbox in Matlab, it took almost three minutes to obtain the final result.

\section{Conclusion}

In this paper, an interval-based methodology was introduced in order to obtain the obstacle-free workspace of two Parallel Manipulators (PMs), namely a 3-RPR PM and 6-DOF Gough-Stewart 
platform. The proposed approach for the obstacle-free workspace and the corresponding results were represented. First, the collisions of proximal segment lines which pass through one point and one interval with the obstacle were investigated. Then a more general case in which the segment line passes through two intervals was used to determine the collisions between the obstacle and medial and distal limbs and also with edges of the EE. Indeed, the collision-free workspace of a 6-UPS spatial parallel manipulator was traced by using the proposed approach. To the best of authors' knowledge, this paper can be regarded as the first study on the collision-free workspace of parallel manipulator using interval analysis and opens an avenue to extend it to more complex parallel manipulators. However, the proposed approach in this paper could be well extended to parallel manipulator with one prismatic joint connecting the base to the EE directly. In fact, due to the limitation of interval analysis, although it is very reliable, it is a computationally intensive approach and in the case of high degree and complex equations, it may lead to very high computing time. Ongoing works deal with the collision detection of limbs between themselves and $\mathrm{EE}$ which is an important issue for the determination of the collision-free workspace of cable-driven parallel robots.

\section{References}

1. J. P. Merlet, Parallel Robots. (Springer, 2006).

2. C. M. Gosselin and M. Jean, "Determination of the workspace of planar parallel manipulators with joint limits," Robot. Auton. Syst. 17(3), 129-138 (1996).

3. O. Bohigas, M. E. Henderson, L. Ros, M. Manubens and J. M. Porta, "Planning singularity-free paths on closed-chain manipulators," IEEE Trans. Robot. 29(4), 888-898, (2013).

4. B. Zi, J. Lin and S. Qian, "Localization, obstacle avoidance planning and control of a cooperative cable parallel robot for multiple mobile cranes," Robot. Comput.-Integr. Manuf. 34, 105-123, (2015).

5. T. Laliberte and C. M. Gosselin, "Efficient Algorithms for the Trajectory Planning of Redundant Manipulators with Obstacle Avoidance," 1994 IEEE International Conference on Robotics and Automation4, (1994), pp. 2044-2049.

6. O. Brock and O. Khatib, "Elastic strips: A framework for motion generation in human environments," Int. J. Robot. Res. 21(12), 1031-1052 (2002).

7. O. Khatib, K. Yokoi, O. Brock, K. Chang and A. Casal, "Robots in human environments: Basic autonomous capabilities," Int. J. Robot. Res. 18(7), 684-696 (1999).

8. A. Komainda and M. Hiller, "Control of Heavy Load Manipulators in Varying Environments," Proceedings of IAARC/IFAC/IEEE International Symposium on Automation and Robotics in Construction, Madrid, Spain (1999) pp. 22-24.

9. P. Jiménez, F. Thomas and C. Torras, “3d collision detection: A survey,” Comput. Graph. 25(2), 269-285 (2001).

10. P. Wenger and P. Chedmail, "On the connectivity of manipulator free workspace," J. Robot. Syst. 8(6), 767-799 (1991).

11. S. Caro, D. Chablat, A. Goldsztejn, D. Ishii and C. Jermann, "A branch and prune algorithm for the computation of generalized aspects of parallel robots," Artif. Intell. 211, 34-50, (2014).

12. M. FarzanehKaloorazi, M. T. Masouleh and S. Caro, "Collision-Free Workspace of 3-rpr Planar Parallel Mechanism Via Interval Analysis," In: Advances in Robot Kinematics. (Springer, 2014) pp. 327-334.

13. B. Bihari, D. Kumar, C. Jha, V. S. Rathore and A. K. Dash, "A geometric approach for the workspace analysis of two symmetric planar parallel manipulators," Robotica, 34(4), 738-763, (2016).

14. R. Moore and F. Bierbaum, Methods and Applications of Interval Analysis. Vol. 2 (Society for Industrial Mathematics, 1979).

15. L. Jaulin, "Set-membership localization with probabilistic errors," Robot. Auton. Syst. 59(6), 489-495 (2011).

16. M. H. F. Kaloorazi, M. T. Masouleh, and S. Caro, "Determining the maximal singularity-free circle or sphere of parallel mechanisms using interval analysis," Robotica 34(1), 135-149, (2016).

17. J. Merlet, "Interval analysis and robotics," Robot. Res., 147-156, (2010).

18. J. P. Merlet, "Solving the forward kinematics of a gough-type parallel manipulator with interval analysis," Int. J. Robot. Res. 23(3), 221-235, (2004).

19. M. Kaloorazi, M. T. Masouleh, and S. Caro, "Interval-Analysis-Based Determination of the SingularityFree Workspace of Gough-Stewart Parallel Robots," Electrical Engineering (ICEE), 2013 21 st Iranian Conference on IEEE, (2013) pp. 1-6.

20. M. H. Farzaneh Kaloorazi, M. Tale Masouleh and S. Caro, "On the Maximal Singularity-free Workspace of Parallel Mechanisms via Interval Analysis," Proceedings of the 2013 MultiBody Dynamics MBD2013, Zagreb, Croatia, (2013).

21. J.-P. Merlet, "Determination of 6d workspaces of gough-type parallel manipulator and comparison between different geometries," Int. J. Robot. Res. 18(9), 902-916 (1999).

22. M.-H. F. Kaloorazi, M. T. Masouleh and S. Caro, "Determination of the maximal singularity-free workspace of 3-dof parallel mechanisms with a constructive geometric approach," Mech. Mach. Theory 84, pp. 25-36 (2015). 
23. A. Pott, D. Franitza and M. Hiller, "Orientation Workspace Verification for Parallel Kinematic Machines with Constant Leg Length," Proceedings of the Mechatronics and Robotics, Aachen, Germany (Sep. 2004) pp. 13-15.

24. J.-P. Merlet, "An Improved Design Algorithm Based on Interval Analysis for Spatial Parallel Manipulator with Specified Workspace," IEEE International Conference on Robotics and Automation, 2001. Proceedings 2001 ICRA., vol. 2. IEEE, (2001), pp. 1289-1294.

25. M. J. Otis, S. Perreault, T.-L. Nguyen-Dang, P. Lambert, M. Gouttefarde, D. Laurendeau and C. Gosselin, "Determination and management of cable interferences between two 6-dof foot platforms in a cable-driven locomotion interface," IEEE Trans. Syst. Man Cybern. A 39(3), 528-544 (2009).

26. M. Gouttefarde, D. Daney and J. Merlet, "Interval-analysis-based determination of the wrench-feasible workspace of parallel cable-driven robots," IEEE Trans. Robot. 27(1), 1-13 (2011).

27. P. Dwyer, "Computation with approximate numbers," pp. 11-34 (1951).

28. T. Sungana, "Theory of Interval Algebra and Application to Numerical Analysis," pp. 29-46, (1958).

29. M. Warmus, "Calculus of approximations," Bull. Acad. Pol. Sci. 4(5), 253-257 (1956).

30. J. Wilkinson, "Turings work at the national physical laboratory and the construction of pilot ace, deuce, and ace," Metropolis et al.[MHR80], pp. 101-114, (1980).

31. R. E. Moore, Interval Analysis. Series in Automatic Computation, (Prentice-Hall, Englewood Cliff, NJ, 1966).

32. E. Hansen and G. Walster, Global Optimization Using Interval Analysis: Revised and Expanded (CRC, 2003, vol. 264).

33. F. Hao and J. Merlet, "Multi-criteria optimal design of parallel manipulators based on interval analysis," Mech. Mach. Theory 40(2), 157-171 (2005).

34. D. Chablat, P. Wenger, F. Majou and J. Merlet, "An interval analysis based study for the design and the comparison of three-degrees-of-freedom parallel kinematic machines," Int. J. Robot. Res. 23(6), 615-624 (2004).

35. R. Rao, A. Asaithambi and S. Agrawal, "Inverse kinematic solution of robot manipulators using interval analysis," J. Mech. Des. 120(1), 147-150 (1998).

36. A. Fusiello, A. Benedetti, M. Farenzena and A. Busti, "Globally convergent autocalibration using interval analysis," IEEE Trans. Pattern Anal. Mach. Intell. 26(12), 1633-1638 (2004).

37. W. Wabinski and H.-J. von Martens, "Time Interval Analysis of Interferometer Signals for Measuring Amplitude and Phase of Vibrations," Second International Conference on Vibration Measurements by Laser Techniques: Advances and Applications. International Society for Optics and Photonics, 1996, pp. 166-177.

38. D. Oetomo, D. Daney, B. Shirinzadeh, and J. Merlet, "Certified Workspace Analysis of 3RRR Planar Parallel Flexure Mechanism," IEEE International Conference on Robotics and Automation (ICRA). IEEE, (2008) pp. 3838-3843.

39. P. Van Hentenryck, D. McAllester and D. Kapur, "Solving polynomial systems using a branch and prune approach,” SIAM J. Numer. Anal. 34(2), 797-827 (1997). 